\title{
Microemulsões e fases líquidas cristalinas como sistemas de liberação de fármacos
}

\author{
Thalita Pedroni Formariz,2, Maria Cristina Cocenza Urban¹, Arnóbio Antônio da Silva Júnior¹, \\ Maria Palmira Daflon Gremião ${ }^{1}$, Anselmo Gomes de Oliveira ${ }^{1 *}$ \\ 'Departamento de Fármacos e Medicamentos, Faculdade de Ciências Farmacêuticas, Universidade Estadual \\ Paulista, ${ }^{2}$ Centro Universitário de Araraquara
}

\footnotetext{
*Correspondência:

A. G. Oliveira

Faculdade de Ciências Farmacêuticas

de Araraquara

Rodovia Araraquara-Jaú, Km 01, 14801 -

902 - Araraquara - SP - Brasil

E-mail: oliveiag@fcfar.unesp.br
}

A mistura de tensoativos com água, em determinadas proporções, na ausência ou na presença de substâncias lipofilicas pode formar diferentes tipos de agregados, entre os quais agregados polimorfos representados pelas microemulsões (ME) e mesofases liotrópicas os cristais líquidos (LC), que estão intimamente ligados com a proporção e a natureza dos componentes da mistura. Nesse trabalho, foi discutido o papel desses sistemas na incorporação de fármacos com diferentes propriedades físico-químicas, influenciando fortemente a liberação, assim como a biodisponibilidade dos fármacos. Aspectos sobre a formação e a caracterização de microemulsões e cristais líquidos também foram discutidos. A análise da literatura indicou que, dependendo da polaridade do fármaco, o efeito da ME ou LC pode ser usado para otimizar o efeito terapêutico por meio do controle da velocidade ou do mecanismo de liberação do fármaco.
Unitermos

- Microemulsões

- Cristais líquidos

- Diagrama de fases

- Sistemas de liberação de fármacos

\section{INTRODUÇÃO}

A formação de microestruturas em soluções aquosas de tensoativos é um fenômeno comum de auto-organização molecular como forma de atingir a estabilidade termodinâmica. Esse fenômeno torna-se a base para a aplicação tecnológica dos tensoativos como sistemas organizados nas ciências biológicas.

Moléculas de tensoativos comumente se auto-agregam na presença de água formando uma rica variedade de estruturas, quando são variados os parâmetros de concentração de tensoativos, presença de sal ou a temperatura. Em soluções diluídas, soluções isotrópicas de agregados micelares podem ser formadas, enquanto que em sistemas tensoativo-solvente em concentrações mais elevadas, fases líquido-cristalinas isotrópicas e anisotrópicas podem existir. Esses agregados tornam-se mais estruturados quando um óleo ou mesmo outros componentes, como outro tensoativo ou um álcool de cadeia média, é adicionado ao sistema tensoativo-água. Dessa forma, emulsões, microemulsões e mesofases liotrópicas de diferentes geometrias podem ser geradas (Ezrahi et al., 1999).

Nos últimos anos a procura por novos sistemas de liberação de fármacos tem sido muito relevante no sentido de se estabelecer alternativas terapêuticas mais eficientes, que possibilitem administrar os fármacos com mais segurança e com efeitos colaterais minimizados (Cera, 2001). 
Uma parte desses trabalhos tem sido dirigida para o estudo de microemulsões (ME) e cristais líquidos, visando ao aumento da solubilidade e da estabilidade de fármacos, a possibilidade de incorporação de fármacos hidrofílicos ou lipofílicos, a capacidade de agir como sistemas reservatórios, a diminuição da toxicidade, assim como a alteração da biodisponibilidade, dependendo do tipo de interação entre fármaco e o sistema de administração (Constantinides et al., 1995; Constantinides, 1995; Brinon et al., 1999; Gabboun et al., 2001).

\section{MICROEMULSÕES}

Muita atenção tem sido dada aos sistemas microe nanoestruturados de tensoativos, por sua capacidade em aumentar a eficácia terapêutica de fármacos, permitindo a redução da dose administrada e minimizando os efeitos colaterais potenciais dos fármacos. Os sistemas micro- e nanoemulsionados são capazes de compartimentalizar fármacos nas gotículas da fase interna, as quais possuem propriedades físico-químicas bastante diferentes das do meio dispersante, induzindo modificações nas propriedades biológicas dos fármacos incorporados (Bhargava et al., 1987). Além disso, esses sistemas melhoram a solubilização de fármacos lipofílicos em água e os protegem contra hidrólise enzimática, além de aumentar o potencial de absorção devido à presença de tensoativo (Cruz, Uckun, 2001).

Microemulsões (ME) podem ser definidas como sistemas termodinamicamente estáveis, isotrópicos, transparentes, de dois líquidos imiscíveis, usualmente água e óleo, estabilizados por um filme de compostos tensoativos, localizados na interface óleo/água (Friberg, Bothorel, 1988; Constantinides et al., 1994, 1995, 1996; Lee et al., 1995; Ho et al., 1996; Lawrence, 1996; Oliveira et al., 1997; Dalmora, Oliveira, 1999; Dalmora et al., 2001). Entretanto, a possibilidade de formar microemulsão depende do balanço entre as propriedades hidrofílicas e lipofílicas do tensoativo, determinada não somente pela sua estrutura química, mas também por outros fatores como temperatura, força iônica e a presença de co-tensoativo. A mistura de tensoativos com equilíbrio hidrófilo-lipófilo adequado proporciona a condição máxima de "solubilização" do óleo e da água (Mital, 1999). Assim, a formação da microemulsão geralmente envolve a combinação de três a cinco componentes, tais como tensoativo, água, óleo e, quando necessário, o co-tensoativo (Lawrence, 1996; Oliveira et al., 1997; Dalmora, Oliveira, 1999; Cruz, Uckun, 2001), sendo que a orientação para sistemas $\mathrm{O} / \mathrm{A}$ ou $\mathrm{A} / \mathrm{O}$ é dependente das propriedades físico-químicas do tensoativo e do óleo, da relação entre as proporções tensoativo/co-tensoativo e entre as proporções água/óleo (Constantinides et al., 1994; Oliveira et al., 1997; Cruz, Uckun, 2001). A principal característica desses sistemas é formar uma emulsão muito fina por homogeneização suave com fase aquosa, cujas dimensões das gotículas da fase interna é da ordem de nanômetros. As ME são superiores às soluções micelares em termos de potencial de solubilização de substâncias, por isso, são usadas para aumentar a solubilização e a absorção de fármacos lipofílicos. Sua estabilidade termodinâmica oferece vantagens sobre as dispersões instáveis, tais como as suspensões e emulsões, possuindo tempo de vida útil muito mais amplo (Constantinides, 1995; Oliveira et al., 1997; Dalmora, Oliveira, 1999; Cruz, Uckun, 2001).

O termo microemulsão foi introduzido por Hoar e Schulman, em 1943, os quais descreveram esses sistemas como transparentes ou translúcidos, obtidos por titulação a partir de uma emulsão comum, a qual apresenta aspecto leitoso e, quando adicionado um álcool de cadeia média, este sistema clarifica (Garti, Aserin, 1996).

O tensoativo pode ser puro, mistura, ou combinação com outros componentes, cuja principal função é a redução da tensão interfacial (Cruz, Uckun, 2001). Para a formação espontânea dos sistemas microemulsionados, a tensão interfacial deve estar próxima de zero. Esse sistema apresenta algumas vantagens tais como transparência, alta estabilidade, fácil preparação e capacidade de incorporar diferentes classes de fármacos com diferentes propriedades físico-químicas (Aboofazeli et al., 2000).

Um sistema de classificação que define os vários equilíbrios existentes entre a microemulsão e as fases aquosa e oleosa foi proposto (Winsor, 1948). Foram estabelecidos quatro tipos de sistemas: 1) Winsor I-É representado pelo equilíbrio entre a fase emulsionada com a fase oleosa em excesso. Por possuir densidade menor que a da emulsão, a fase oleosa posiciona-se acima da emulsão; 2) Winsor II-Representa o equilíbrio entre a fase emulsionada e a fase aquosa em excesso. Por possuir densidade menor que a da fase aquosa, a emulsão posiciona-se na parte superior à fase aquosa; 3 ) Winsor III- Existem três fases em equilíbrio, óleo, emulsão e água, em que o óleo é a fase superior, a emulsão a fase intermediária e a água, a fase inferior; 4) Winsor IV-É um sistema em que apenas existe a fase microemulsão, isto é, um sistema visualmente monofásico. A Figura 1 mostra detalhes do sistema de Winsor.

A natureza e estrutura do tensoativo, co-tensoativo e óleo são fatores essenciais na formulação de sistemas microemulsionados. A construção de diagramas de fase 


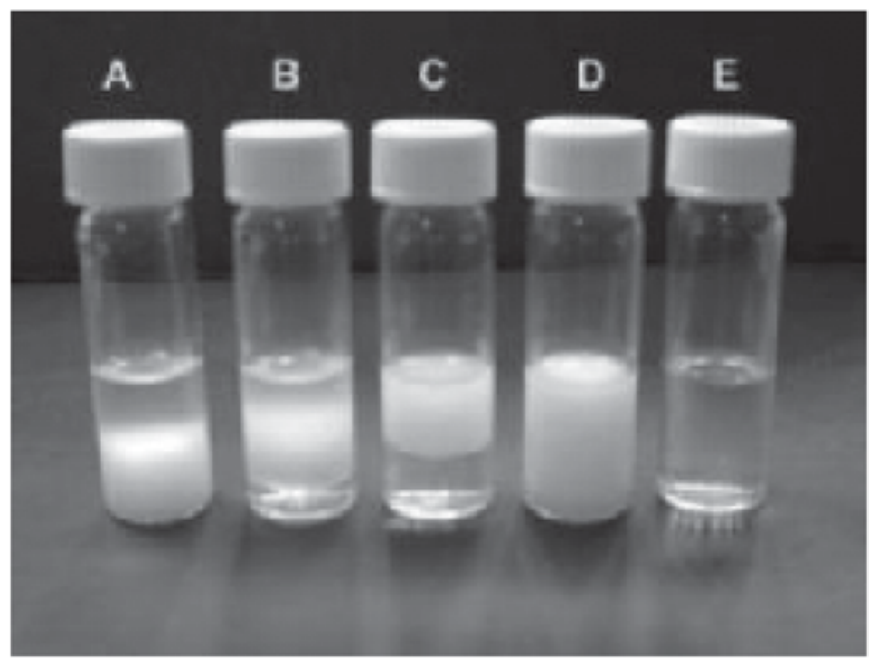

FIGURA 1 - Representação da classificação de Winsor. A) Winsor I. B) Winsor III. C) Winsor II. D) Emulsão homogênea. E) Winsor IV.

pode ser uma ferramenta fundamental para caracterizar em que condições experimentais as ME existem e em que proporções dos componentes outras estruturas podem estar presentes. A partir desses dados, pode-se selecionar a região do diagrama de fases que mais convenientemente represente a condição mais apropriada para que o fármaco seja incorporado (Formariz, 2004).

Os diagramas pseudoternários podem ser obtidos a partir de dados de titulação ou pela preparação de amplo número de amostras com diferentes proporções dos componentes. A vantagem do primeiro método é que este pode ser usado para estudar amplo número de amostras de diferentes composições de maneira rápida (Bhargava et al., 1987; Lawrence, Rees, 2000). Normalmente, os sistemas microemulsionados podem ser diferenciados visualmente dos outros sistemas, uma vez que os demais sistemas apresentam como emulsões líquidas opacas, emulsões géis opacas, representadas por sistemas de viscosidade elevada, ou com separação de fases. Já os sistemas microemulsionados líquidos são caracterizados como sistemas translúcidos ou opticamente transparentes (Formariz, 2004).

A literatura mostra que a adição de um cotensoativo diminui o tamanho das gotículas e amplia as regiões de microemulsões (Kuneida et al., 1999). Dessa forma, o tamanho da gotícula é fortemente afetado pela mistura de tensoativo no sistema, havendo tendência à diminuição do tamanho das gotículas com o aumento da concentração de tensoativo, gerando sistema opticamente transparente ou translúcido (Lunberg et al., 1996). Isso ocorre porque a mistura de tensoativo com um co-tensoativo é mais eficiente em reduzir a tensão interfacial entre óleo-água, proporcionado a redução máxima do tamanho das gotículas da fase interna (Trotta et al., 2002).

Microemulsões podem formar várias estruturas, tais como gotículas de óleo em água, gotículas de água em óleo, misturas randômicas bicontínuas, gotículas ordenadas e misturas lamelares com ampla faixa de equilíbrio entre elas e com excesso de fase oleosa e/ou aquosa, sendo que a formação dessas estruturas depende do tensoativo, do co-tensoativo e da natureza do óleo. Portanto, a escolha adequada e as concentrações desses componentes tornam-se extremamente importantes para a orientação desses sistemas (Sirotti et al., 2002). Devese ressaltar que tensoativos não-iônicos e zwiteriônicos são mais aceitáveis para aplicações farmacêuticas e formulações microemulsionadas porque são menos afetados pelas mudanças de $\mathrm{pH}$ e força iônica e contornam problemas de toxicidade (Cruz, Uckun, 2001).

Do ponto de vista microestrutural, as ME podem ser do tipo água em óleo $(\mathrm{A} / \mathrm{O})$, óleo em água $(\mathrm{O} / \mathrm{A})$ ou estruturas bicontínuas (Cruz, Uckun, 2001) (Figura 2). Nas microemulsões do tipo A/O, o componente hidrofílico é disperso na forma de gotículas coloidais no componente lipofílico. Já nas ME do tipo O/A, o componente lipofílico é disperso na forma de gotículas coloidais no componente hidrofílico (Mo et al., 2000; Cruz, Uckun, 2001) e ambas podem ser invertidas de $\mathrm{A} / \mathrm{O}$ para $\mathrm{O} / \mathrm{A}$ ou vice-versa ao variar as condições de emulsificação (Mo et al., 2000). Em relação ao último tipo de sistema microemulsionado, os componentes hidrofílicos e lipofílicos formam microemulsão com estrutura bicontínua, com canais adjacentes alongados com gotículas na faixa de 1-100 nm (Cruz, Uckun, 2001), como uma rede de tubos aquosos em matriz oleosa ou rede de tubos oleosos em matriz aquosa (Mo et al., 2000), e contendo volumes relativos aproximadamente iguais entre a fase aquosa e oleosa (Lawrence, Rees, 2000; Mo et al., 2000; Cruz, Uckun, 2001). Se a microemulsão apresentar tal estrutura, essa poderá ser utilizada como sistema transportador de fármacos hidro e lipossolúveis (Cruz, Uckun, 2001).

Sistemas microemulsionados são geralmente caracterizados como agregados esféricos e com diâmetros menores que $1400 \AA$, geralmente de $100 \AA$ (Langevin, 1988; Oliveira, Scarpa, 2001; Oliveira et al., 2004). Essa afirmação baseia-se no fato de, em um sistema coloidal, se o diâmetro das gotículas for menor do que $1 / 4$ do comprimento de onda da luz incidente, as partículas não espalharão luz, resultando em sistema opticamente transparente (Rosano, 1974; Oliveira, Scarpa, 2001). Porém, quando esses sistemas são caracterizados por técnicas de espalhamento de raios-X a baixo ângulo (SAXS) e de espalhamento de nêutrons a baixo ângulo (SANS), indi- 


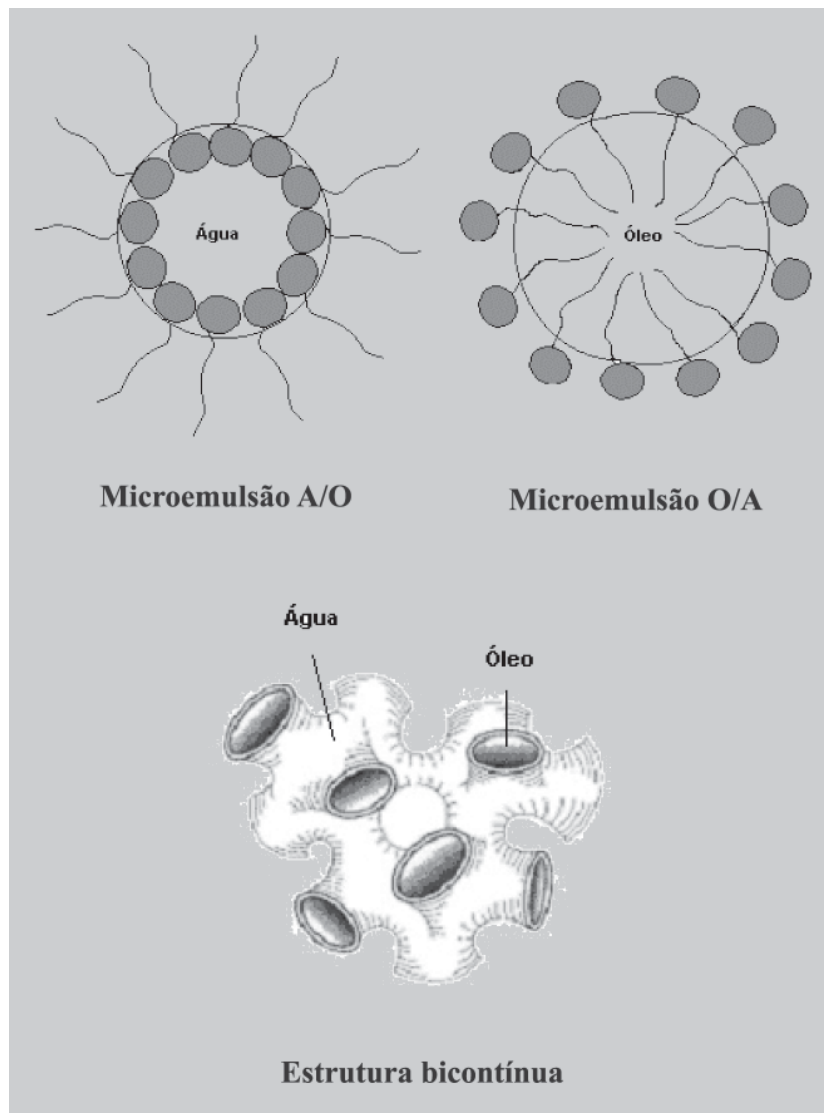

FIGURA 2 - Representação esquemática da organização das microemulsões (Castillo, 2002).

cam que o óleo e a água não são dispersões moleculares e, sim, misturas mais grosseiras (Lindman, Friberg, 1999).

Os meios dispersos apresentam a propriedade de espalhar as ondas eletromagnéticas (como a luz), que venham atravessá-los. Este fenômeno depende do tamanho das gotículas que compõem o sistema disperso e do comprimento de onda utilizado (Silva, 2001). A diafaneidade de um meio pode ser utilizada para identificar as ME e estimar de forma grosseira o tamanho das gotículas. A tabela a seguir

TABELA I - Tamanho de agregado (gotículas dispersas) e diafaneidade do meio (Silva, 2001)

\begin{tabular}{|c|c|c|}
\hline Agregados & $\begin{array}{l}\text { Diâmetro das } \\
\text { gotículas (Å) }\end{array}$ & Diafaneidade \\
\hline Micelas & $35-75$ & Transparentes \\
\hline Microemulsões & $100-2000$ & $\begin{array}{c}\text { Transparentes/ } \\
\text { Translúcidas }\end{array}$ \\
\hline Emulsões & $2000-100000$ & $\begin{array}{c}\text { Opaca/ } \\
\text { Esbranquiçada }\end{array}$ \\
\hline $\begin{array}{l}\text { Unidade de } \\
\text { resolução visual }\end{array}$ & 500000 & $\begin{array}{l}\text { Discretos } \\
\text { agregados }\end{array}$ \\
\hline
\end{tabular}

relaciona o diâmetro de alguns agregados e como estes se apresentam, quanto à dispersão da luz.

O fenômeno de dispersão da luz pelas microemulsões pode ser avaliado de maneira eficiente através da técnica de "Light Scattering", a qual fornece informações diretas sobre o movimento translacional das gotículas da ME e permite o cálculo do tamanho da gotícula através de relações empíricas adequadas (Aboofazeli et al., 2000). Nota-se, no entanto, que se o sistema é concentrado, ou seja, quando a fase dispersa estiver em elevadas concentrações, a interpretação torna-se dificultada em razão das interações intergotículas. Para suprimir a interação entre gotículas a diluição do sistema microemulsionado com a fase dispersante é necessária (Hou et al., 1988; Lawrence, Rees, 2000; Aboofazeli et al., 2000).

Sistemas com elevada viscosidade, também podem conter erros experimentais no tamanho das gotículas (Aboofazeli et al., 2000), em razão de que a técnica de determinação é fundamentada no movimento Browniano das gotículas em dispersão, sendo o coeficiente de difusão das gotículas no meio o principal parâmetro levado em consideração (Orthaber, Glatter, 2000).

A determinação da densidade, viscosidade, espalhamento de luz, índice de refração são importantes como técnicas de caracterização física (Constantinides et al., 1995). A literatura refere que técnicas experimentais diferentes podem ser usadas para obter informações sobre a dinâmica e estrutura das ME (Kahlweit et al., 1987). Diferenças de viscosidade podem diferenciar sistemas microemulsionados comuns de sistemas géis. Gotículas de fase interna com tamanhos extremamente pequenos são característica de sistemas termodinamicamente estáveis se esses formarem espontaneamente, mas o aumento do diâmetro com o volume de fase interna, geralmente, leva à modificação da microestrutura interna do sistema, na qual a estrutura esférica das gotículas modifica-se para fases hexagonais e contínuas (Constantinides et. al., 1995; Oliveira, 1997; Avramiotis et al., 1997).

A reologia consiste no estudo do escoamento ou deformação do material em estudo quando submetido a uma tensão (Wood, 1986). O método é aplicável na caracterização de sistemas micro e emulsionados, pois o comportamento do fluído está relacionado com o tipo e grau de organização do sistema. Também podem ser empregadas para checar a qualidade durante o processo de produção, além de permitir o estudo do efeito de aditivos na formulação (Scott, 2000).

Os sistemas microemulsionados e emulsionados se comportam na maioria das vezes, como sistemas nãoNewtonianos, os quais se caracterizam por mudança na 
viscosidade com o aumento da velocidade de cisalhamento, em que a relação entre tensão e velocidade de cisalhamento não é constante e os gráficos apresentam-se como uma curva (Wood, 1986; Scott, 2000). Freqüentemente, as ME também estão associadas à tixotropia (Cera, 2001; Formariz, 2004).

Correa e colaboradores (2005) mostraram que a viscosidade aparente de sistemas microemulsionados e emulsionados é dependente do volume de fase e da natureza da fase oleosa, visto que o aumento do volume de fase interna oleosa provocou aumento da viscosidade aparente e que microemulsões com estruturas bicontínuas apresentam valores de viscosidade mais baixos.

A caracterização físico-química dos sistemas emulsionados e microemulsionados, principalmente por estudos de viscosidade e determinação do tamanho das gotículas, pode auxiliar na interpretação dos experimentos de liberação in vitro e in vivo. O diâmetro das gotículas da fase interna das emulsões e microemulsões assim como a viscosidade do sistema estão diretamente relacionados com a velocidade de liberação in vitro e com o processo de difusão in vivo, facilitando ou dificultando a fração disponível de fármaco por unidade de tempo (Correa, 2005).

\section{Vias de administração}

Dependendo de sua composição, as microemulsões não apresentam restrições em relação às vias de administração, podendo ser aplicadas pela via oral, ocular (Garti, Aserin, 1996), tópica, dérmica (Cruz, Uckun, 2001), transdérmica, parenteral (Garti, Aserin, 1996; Li et al., 2002), pulmonar, vaginal, retal (Cruz, Uckun, 2001) e nasal (Li et al., 2002).

\section{Microemulsões como Sistemas de Liberação de Fármacos}

Nos últimos anos, tem aumentado o interesse na aplicação de microemulsões como sistema de liberação de fármacos, já que esses agregados apresentam-se descritos na literatura como sistemas reservatórios e permitem liberação lenta do fármaco, a qual proporciona efeito prolongado, evitando atingir concentração plasmática acima do necessário (Dalmora et al., 2001).

Dispersões coloidais óleo em água representam interessante perspectiva para o desenvolvimento de formulações, pois estas são utilizadas como veículos para liberação de fármacos pouco solúveis em água, especialmente quando administrados pela via parenteral. Porém, esses sistemas requerem alguns cuidados especiais, tais como, esterilidade, apirogenicidade, isotonicidade, atoxicidade e estabilidade. Além disso, o tamanho das gotículas da fase dispersa deve ser menor do que $1 \mathrm{~mm}$, pois gotículas de óleo maiores podem causar embolia. As microemulsões obedecem esse último requisito (Trotta, 1999).

Microemulsões lipídicas do tipo O/A são alternativas interessantes como sistemas de liberação de fármacos (Figura 3). A estrutura básica da microemulsão lipídica é um lipídio neutro na região interna, como, por exemplo, um triglicerídio líquido, estabilizado por lipídios anfifílicos, como os fosfolipídios. Esse sistema pode solubilizar quantidade considerável de fármacos lipossolúveis no domínio hidrofóbico da fase interna oleosa e, portanto apresenta potencial de aplicação terapêutico como transportadores de fármacos lipossolúveis (Lundberg et al., 1996).

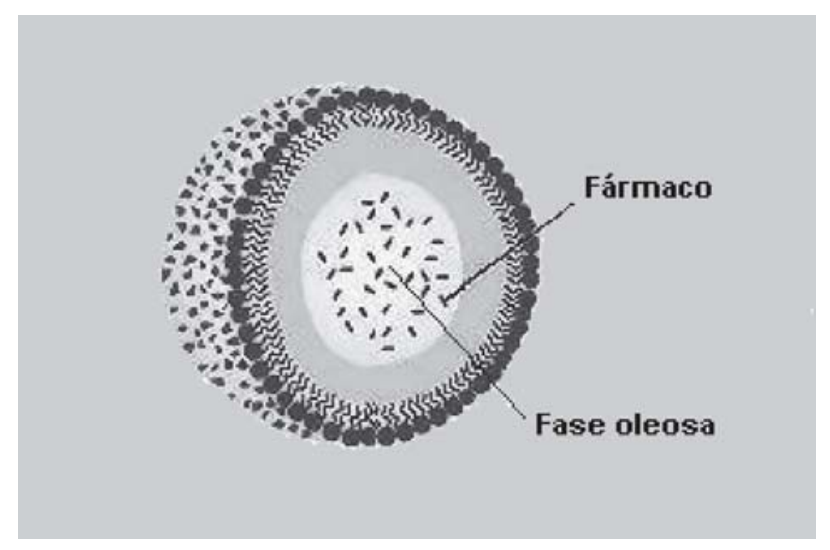

FIGURA 3 - Microemulsão do tipo O/A (Oliveira et al., 2004).

A literatura relata que fármacos parcialmente hidrofílicos solubilizados em sistemas microemulsionados, podem particionar entre a fase contínua, interior da ME e na região interfacial. Portanto, a região interfacial pode não consistir inteiramente de compostos tensoativos. Além disso, a literatura também mostra que o aumento da solubilidade do fármaco nas gotículas dispersas de óleo está relacionado com a partição favorável do mesmo nesta fase e o sistema terá característica de reservatório, apresentando liberação prolongada do fármaco. Essa liberação acontece devido à difusão lenta e contínua de fármaco das gotículas de óleo para a fase contínua (Garti, Aserin, 1996).

As ME do tipo água em óleo $(\mathrm{A} / \mathrm{O})$ também têm sido descritas como sistemas transportadores de fármacos hidrofílicos, principalmente para liberação de fármacos por via oral (Ritschel, 1991; Sarciaux et al., 1995; Park, Kim, 1999) e intramuscular (Gasco et al., 1990; Park, Kim, 1999). No caso de microemulsões do tipo A/O, a inversão de fase é uma propriedade interessante, que resulta em 
microemulsões do tipo $\mathrm{O} / \mathrm{A}$, e estas podem ser usadas para liberação de fármacos via oral (Park, Kim, 1999; Wargaftigi, 2000). A literatura relata que ME A/O, podem ser facilmente invertidas em $\mathrm{ME} \mathrm{O/A} \mathrm{e/ou} \mathrm{emulsões}$ múltiplas do tipo água-óleo-água (A/O/A), quando estas são diluídas com excesso de fase aquosa. As microemulsões do tipo $\mathrm{O} / \mathrm{A}$ apresentam muitas vantagens como sistema de liberação de fármacos, pois estes melhoram a solubilização de fármacos lipofílicos, tornando possível o uso parenteral e permitindo assim, a produção em larga escala industrial sem o uso de energia para a homogeneização (Park, Kim, 1999). Por outro lado, estudos experimentais mostram a melhora na absorção oral, quando se utiliza sistema microemulsionado. Esta melhora é devida à incorporação do fármaco nas gotículas das microemulsões $(\mathrm{O} / \mathrm{A})$ e, também, ao aumento específico da área superficial, resultando no intenso contato das gotículas com o trato gastrintestinal. Outro fator importante é o tamanho e a polaridade das gotículas das ME resultante do balanço hidrofílico e lipofílico do tensoativo, pois a variação dessa polaridade influencia a incorporação do fármaco e do tensoativo na interface, alterando a propriedade de liberação do fármaco (Itoh et al., 2002).

Microemulsões também apresentam grande potencial como veículos de liberação de fármacos lipofílicos via intravaginal e retal, tais como, microbicidas, esteróides e hormônios, porque aumentam a capacidade de solubilização de fármacos, aumentam absorção e melhoram a eficiência clínica. Entretanto, o uso desse sistema para administração intravaginal e retal impôs rigorosas exigências no que diz respeito à toxicidade e à biodisponibilidade da formulação, uma vez que, para esse sistema ser considerado adequado para aplicação intravaginal, deve apresentar gotículas com diâmetros de 30-80 nm e os excipientes farmacêuticos usados devem contornar problemas de toxicidade (Cruz, Uckun, 2001).

Cortesi e colaboradores (1997) utilizaram sistemas microemulsionados para solubilizar a camptotencina, alcalóide citotóxico bastante utilizado no tratamento de paciente com câncer, em especial, de pulmão, ovário e mama. A solubilidade desse fármaco é muito baixa, cerca de $1,3 \mathrm{mg} / \mathrm{mL}$, e isto causa sério inconveniente aos pacientes, uma vez que este medicamento só pode ser administrado através de infusões contínuas ou múltiplas injeções diárias, o que resulta em vários efeitos adversos: neutropenia, trombocitopenia, anemia, alopecia, náusea, vômitos, diarréia e erupções cutâneas. Os sistemas microemulsionados aumentaram consideravelmente a solubilidade da camptotencina, pois esta chegou a $500 \mathrm{mg} / \mathrm{mL}$. Esse efeito associado à maior penetração em membranas biológicas pode contribuir para o aumento da absorção gastrointestinal dos alcalóides e, então, facilitar a administração oral (Cortesi, Nastruzzi, 1999; Formariz et al., 2004).

Trabalhos desenvolvidos em nosso laboratório mostram que ME aumentam significativamente a solubilidade de antiinflamatórios não-esteróides (Dalmora, 1996; Correa, 1996; Dalmora, Oliveira, 1999; Oliveira et al., 2004) e funcionam como sistemas reservatórios, proporcionando atividade terapêutica mais intensa e por tempo prolongado. Também foi demonstrado que ME constituem veículo muito mais eficiente que a solução aquosa para administração de vacinas de DNA por via oral (Wargaftigi, 2000; Oliveira et al., 2004). Nesse contexto, os sistemas coloidais de veiculação de fármacos, particularmente as ME, também têm sido utilizados como estratégia para aumentar o índice terapêutico dos quimioterápicos antineoplásicos, com a conseqüente redução dos efeitos adversos (Maranhão et al., 1995; 1996; Mitra et al., 2001; Owens et al., 2001; Formariz, 2004).

A literatura mostra que quando os fármacos são incorporados em sistemas microemulsionados, o transporte é maior do que em cristais líquidos. Esse fato pode ser atribuído às diferenças estruturais desses sistemas, os quais restringem o movimento das moléculas do fármaco, $\mathrm{e}$ às interações soluto-solvente no ambiente anisotrópico $\mathrm{e}$ isotrópico, visto que os cristais líquidos apresentam alta energia de ativação de difusão de fármacos e baixa difusividade de fármaco (Gabboun et al., 2001).

A análise detalhada dos diagramas de fase mostra que a mistura dos componentes das microemulsões em diferentes proporções podem gerar vários tipo de agregados nos limites desses diagramas. Assim, no domínio dos agregados polimorfos pode-se detectar a existência das emulsões, microemulsões e cristais líquidos (Ezrahi et al., 1999). Nessas várias formas de agregação, microemulsões e cristais líquidos podem influenciar diferentemente a velocidade de liberação dos fármacos (Gabboun et al., 2001).

\section{CRISTAIS LÍQUIDOS}

Cristais líquidos constituem uma fase distinta de estruturas condensadas, cujas características físicas os posicionam entre sólidos e fundidos, com parcial ordem/ desordem das espécies atômicas. Por essa razão, são também chamados de mesofases, em que o prefixo grego meso significa intermediário. Materiais que formam cristais líquidos pela adição de solventes são chamados cristais líquidos liotrópicos, enquanto cristais líquidos termotrópicos têm sua estabilidade dependente da temperatura (Hyde, 2001).

Mesofases liotrópicas contêm, no mínimo, dois componentes: o componente orgânico, por exemplo, tensoativo e seu solvente. A porção orgânica deve exibir certa complexidade química, do contrário, o solvente iria simplesmen- 
te solubilizar a molécula, originando soluções moleculares de moléculas dispersas e desordenadas, e certamente não seriam formados cristais líquidos. A adição de um solvente, tal como a água, irá hidratar seletivamente a porção hidrofílica das moléculas de tensoativo, evitando as regiões hidrofóbicas (Hyde, 2001).

O fenômeno essencial comum aos estados cristalinos é a presença de orientação ordenada das interfaces. A fim de caracterizar o comportamento de fase de sistemas líquido-cristalinos, é necessário conhecer a existência de outras mesofases, as quais não são (stricto sensu) cristais líquidos, como microemulsões isotrópicas, mesofases esponjosas e emulsões, cujas interfaces não possuem qualquer tipo de ordem orientacional (Ezrahi et al., 1999; Hyde, 2001).

Mesofases liotrópicas podem ser consideradas micelas ordenadas com arranjo molecular caracterizado por regiões hidrofóbicas e hidrofílicas alternadas. Conforme o aumento da concentração de tensoativo, diferentes formas líquido-cristalinas podem ser formadas, como lamelares, hexagonais e cúbicas (Gabboun et al., 2001).

A fase lamelar (designada $\mathrm{L}_{\mathrm{a}}$ ) é formada por camadas paralelas e planas de bicamadas de tensoativo separadas por camadas de solvente, formando rede unidimensional (Figura 4-A). Já na fase hexagonal, os agregados são formados pelo arranjo de cilindros longos, originando estruturas bidimensionais. $\mathrm{Na}$ fase $\mathrm{H}_{\mathrm{I}}$ (fase normal), as moléculas do tensoativo se agrupam em micelas cilíndricas circulares, com água preenchendo o volume entre os cilindros, enquanto que na fase $\mathrm{H}_{\mathrm{II}}$ (fase reversa), os cilindros contêm canais de água circundados pelas cabeças polares do tensoativo e a porção oleosa localizada ao redor dos cilindros (Figura 4-2) (Ezrahi et al., 1999).

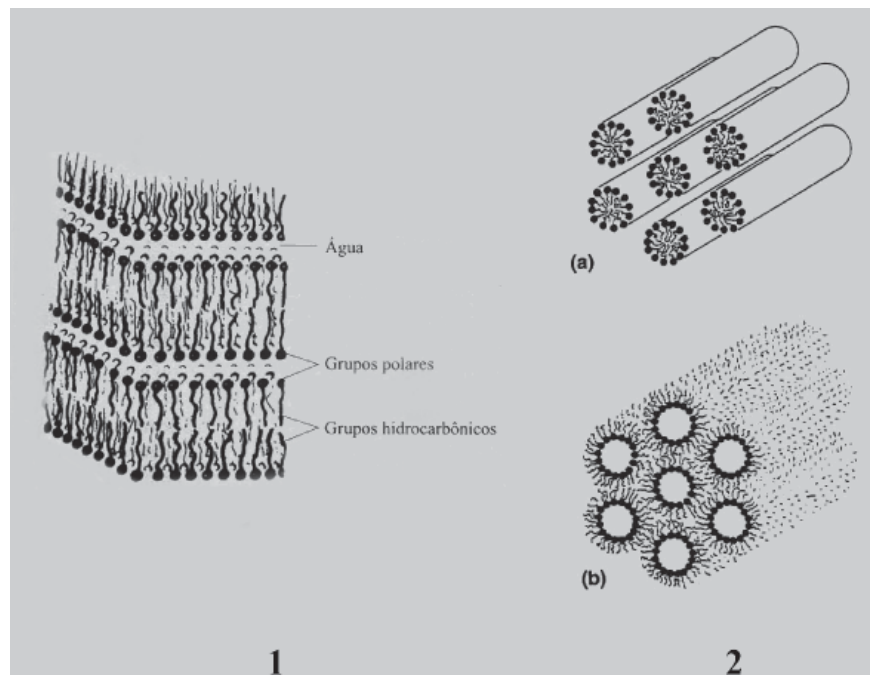

FIGURA 4 - Ilustração esquemática (1) fase lamelar e (2.a) fase hexagonal (2.b) fase hexagonal reversa (Ezrahi et al., 1999).
Fases cúbicas liotrópicas (Figura 5) apresentam estruturas mais complicadas e visualizadas com maior dificuldade que as outras. Quase todas as fases fluidas tridimensionais observadas são de simetria cúbica, apesar das romboédricas, tetragonais e ortorrômbicas de topologia inversa também serem detectadas em alguns sistemas (Ezrahi et al., 1999).

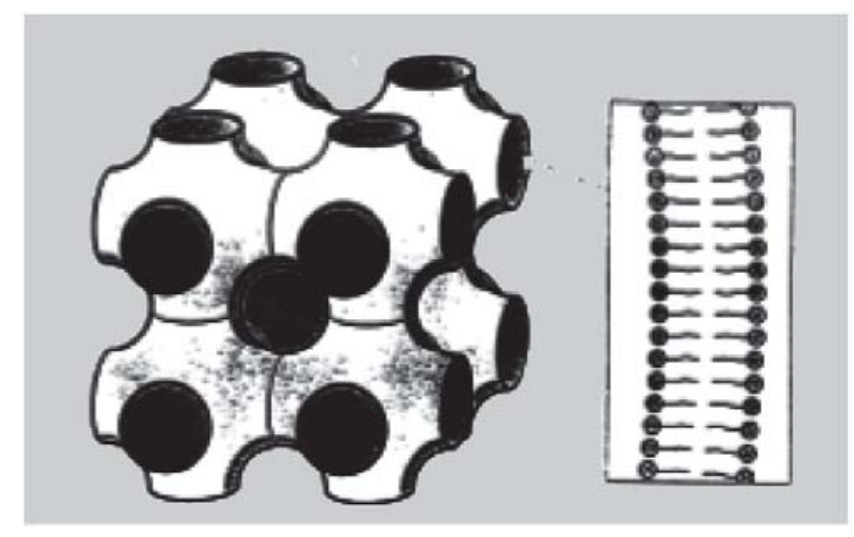

FIGURA 5 - Estrutura da fase cúbica de monoleoato de glicerila-água em três dimensões com ampliação mostrando bicamada lipídica (Shah et al., 2001).

Um dos maiores problemas associados ao desenvolvimento de sistemas de liberação de fármacos é, sem dúvida, a escolha dos constituintes. Essa escolha deve ser bastante criteriosa e deve atender alguns requisitos, como não-toxicidade, não-irritabilidade, capacidade de solubilização do fármaco a ser incorporado no sistema, assim como capacidade de formar o sistema desejado (Lawrence, Rees, 2000; Kreilgaard, 2002).

As mesofases líquido-cristalinas são geralmente formadas pela presença de tensoativos da classe dos polioxietilenos, polímeros e lipídeos, como mono e triglicerídeos. Dentre os lipídios mais utilizados na composição desses sistemas, estão a monoleato de glicerila (monoleína) e o monolinoleato de glicerila (Norling et al., 1992; Carr et al., 1997; Chang, Bodmeier, 1997; Lee, Kellaway, 2000; Urban, 2004). Dependendo da temperatura e da concentração dessas moléculas em água, formam-se agregados bem estruturados, tais como fases cúbicas, hexagonais ou lamelares (Brinon et al., 1999; Farkas et al., 2001; Gabboun et al., 2001).

\section{Caracterização físico-química}

As mesofases líquido-cristalinas podem ser grosseiramente caracterizadas verificando-se a viscosidade. A fase lamelar geralmente apresenta-se como líquido viscoso e a fase hexagonal tem a viscosidade semelhante à de um 
gel, enquanto que a viscosidade da fase cúbica é extremamente elevada (Gabboun et al., 2001; Hyde, 2001). Medidas de reologia são utilizadas a fim de se determinar a viscosidade dinâmica, obtendo-se como resultado a curva de viscosidade em relação ao fluxo e à visco-elasticidade, por meio dos parâmetros módulo de armazenagem (G') e módulo de perda (G”), obtendo-se, neste caso, informação sobre a aparência das amostras (Schramm, 2002).

Uma das maneiras de classificar as fases líquidocristalinas é determinar sua isotropia óptica, através da técnica de microscopia de luz polarizada. Sob um plano de luz polarizada, a amostra é anisotrópica se for capaz de desviar o plano da luz incidente e isotrópica se não desviar a luz. Mesofases lamelares e hexagonais são anisotrópicas, enquanto as cúbicas são isotrópicas (Norling et al., 1992; Brinon et al., 1999; Hyde, 2001).

Para análises mais aprofundadas da estrutura das mesofases, disposição das interfaces, tamanho de micelas, diversas técnicas mais sofisticadas são comumente utilizadas, como microscopia eletrônica de criofractura (Ezrahi et al., 1999), difração de nêutrons (Hyde, 2001), espalhamento de raios-X a baixo ângulo (SAXS), espalhamento de nêutrons a baixo ângulo (SANS) (Farkas et al., 2001) e calorimetria exploratória diferencial (DSC) (Farkas et al., 2000).

\section{Vias de administração}

As mesofases líquido-cristalinas podem ser administradas através de diversas vias, como oral, retal, subcutânea, tópica, transdérmica, intramuscular e endovenosa (Miyazaki et al., 1986; Wyatt, Dorschel, 1992; Carr et al., 1997; Brinon et al., 1999; Lee, Kellaway, 2000; Urban, 2004).

Para administração oral, o sistema contendo o fármaco deve ser fundido e colocado em cápsulas, de modo a ser ingerido e, então, transformado in situ na fase cúbica após contato com os fluidos gastrintestinais (Wyatt, Dorschel, 1992).

Já para a administração parenteral, a fase cúbica e o próprio monoglicerídeo apresentam-se muito viscosos para administração direta, tanto intramuscular como subcutânea. Dessa forma, é necessário que o sistema de liberação apresente baixa viscosidade para que possa ser injetado e se transforme, posteriormente, em fase cúbica (Chang, Bodmeier, 1998).

\section{Cristais Líquidos como Sistemas de Liberação de Fármacos}

Esses sistemas podem ser considerados como micelas ordenadas com arranjo molecular caracterizado por regiões hidrofílicas e lipofílicas alternadas. Os fármacos, quando incorporados nesses sistemas, poderão residir nessas duas regiões e também na bicamada de tensoativo da mesofase, dependendo da solubilidade do fármaco (Muller-Goymann, Frank, 1986).

As interações fármaco-sistema desempenham importante papel no controle da liberação. Entretanto, a razão de liberação de fármacos incorporados em sistemas líquidocristalinos dependerá da estrutura da mesofase assim como das características físico-químicas do fármaco. Essas propriedades tornam possível a utilização de cristais líquidos como veículos transportadores de fármacos, os quais podem ser capazes de controlar a liberação das substâncias neles incorporados (Gabboun et al., 2001).

Como conseqüência da interação fármaco-solvente, sob certas condições, os fármacos solubilizados podem induzir mudanças no empacotamento molecular do sistema. Este efeito pode influenciar a razão de liberação in vitro (Muller-Goymann, Frank, 1986; Ibrahim et al., 1993; Farkas et al., 2001).

Miyazaki e colaboradores (1986) utilizaram géis de Pluronic F-127, os quais se apresentaram como cristais líquidos isotrópicos de alta viscosidade, para liberação controlada de fármacos pelas vias tópica e retal. Nestes casos, as fases géis líquido-cristalinas apresentaram-se como sistemas reservatórios para a indometacina, que foi liberada por difusão através dos canais de água da matriz do gel.

Além de afetar a liberação do fármaco, a mesofase pode alterar a direção do seu movimento, assim como aumentar sua solubilidade, em função das regiões de diferentes polaridades existentes nesses sistemas (Farkas et al., 2001).

Entre as inúmeras vantagens apresentadas pelos cristais líquidos como veículos para administração de fármacos, a literatura também tem demonstrado propriedades bioadesivas das fases cúbicas e lamelares, embora o mecanismo de bioadesão ainda não esteja completamente elucidado (Engström et al., 1995; Nielsen et al., 1998).

A fase cúbica exerce ação protetora contra degradação enzimática de fármacos peptídicos e o aumento da estabilidade química de compostos contendo pontes amídicas (Nielsen et al., 1998).

Gabboun e colaboradores (2001) estudaram a liberação de ácido salicílico, diclofenaco ácido e seus sais incorporados em sistemas estruturados liotrópicos anisotrópicos (fases lamelar e hexagonal) e isotrópicos (sistema micelar) através da pele de ratos sem pêlo. As mesofases foram preparadas a partir da mistura do tensoativo polioxietileno (20) hexadecil éter em água ou tampão de ácido clorídrico $\mathrm{pH}$ 1. Os resultados indicaram que o transporte de ácido salicílico foi maior quando incorporado em sistemas isotrópicos micelares do que em anisotrópicos (alta energia de ativação de difusão de 
fármacos e baixa difusividade de fármacos), e que o transporte de diclofenaco ácido e seus sais através da pele foi cerca de 30 vezes menor que o do ácido salicílico. Esses resultados podem ser atribuídos às diferenças estruturais desses compostos, que restringem o movimento das moléculas do fármaco, e às interações soluto-solvente no ambiente anisotrópico e isotrópico, causando a liberação do diclofenaco e seus sais em diferentes razões.

\section{CONCLUSÕES}

Microemulsões e cristais líquidos podem modificar profundamente a velocidade de liberação de fármacos, oferecendo benefícios que incluem o aumento da solubilidade e absorção e controle da biodisponibilidade de fármacos. Como sistemas reservatórios podem alterar os parâmetros farmacocinéticos, diminuindo a toxicidade e aumentando a eficácia clínica de fármacos.

\section{ABSTRACT \\ Microemulsion and liquid cristals as drug delivery systems}

Depending on the proportions, the mixture of surfactants with water, in the presence or absence of oil, may form different types of supramolecular aggregates. Among these, polymorphic structures represented by microemulsions (ME) and lyotropic mesophases-liquid crystals $(L C)$, closely related with the nature and the proportion of the constituents, can exist. In this work, the role of these systems in the incorporation of drugs with different physicochemical properties, influencing strongly the drug release as well as the bioavailability of the drugs, was discussed. Aspects about the formation and the characterization of $M E$ and $L C$ were also discussed. The analysis of the literature indicate that depending on the drug polarity the effect of ME or LC can be used to optimizes therapeutic effects through the control of rates and the drug release mechanisms.

UNITERMS: Microemulsion. Liquid crystals. Phase diagram. Drug delivery systems.

\section{REFERÊNCIAS BIBLIOGRÁFICAS}

ABOOFAZELI, R.; BARLOW, D.; LAWRENCE, M.J. Particle size analysis of concentrated Phospholipid microemulsions II. Photon correlation spectroscopy. APPS PharmSci., Arlington, v.2, n.3, E.19, 2000.
AVRAMIOTIS, S.; BECKIARI, V.; LIANOS, P.; XENASKIS, A. Structural and dynamic properties of lecithin-alcohol based w/o microemulsions: A luminescence quenching study. J. Coll. Int. Sci., London, v.195, p.326-331, 1997.

BHARGAVA, H.N.; NARURKAR, A.; LIEB, L.M. Using microemulsions for drug delivery. Pharm. Technol., Duluth, v.3, p.46-54, 1987.

BRINON, L.; GEIGER, S.; ALARD, V.; DOUCET, J.; TRANCHANT, J-T., COUARRAZE, G. Percutaneous absorption of sunscreens form liquid crystalline phases. $J$. Control. Rel., Arlington, v.60, p.67-76, 1999.

CAAR, M.G.; CORISH, J.; CORRIGAN, O.I. Drug delivery from a crystalline base across Visking and human stratum corneum. Int. J. Pharm., Amsterdam, v.157, p.35-42, 1997.

CASTILLO, R. Surfactants and Microemulsions, 2002. [On-line] Disponível em: http:/www.fisica.unam.mx/ liquids/tutorials/microemulsions.htm Acesso em: 15 jun. 2004.

CERA, R.F.L. Estudo da incorporação do diclofenaco sódico com microemulsão lipídicas: formulação e liberação in vitro. Araraquara, 2001. 145p. [Dissertação de Mestrado. Faculdade de Ciências Farmacêuticas, Universidade Estadual Paulista].

CHANG, C-M.; BODMEIER, R. Low viscosity monoglyceride-based drug delivery systems transforming into a highly viscous cubic phase. Int. J. Pharm., Amsterdam, v.173, p.51-60, 1998.

CONSTANTINIDES, P.P.; WELZEL, G.; ELLENS, H.; SMITH, P.L.; STURGIS, S.; YIV, S.H.; OWEN, A.J. Water in oil microemulsions containing medium-chain fatty acid/salts: formulation and intestinal absorption enhancement evaluation. Pharm. Res., Arlington, v.13, p.210-215, 1996.

CONSTANTINIDES, P.P.; LANCASTER, C.M.; MARCELLO, J.; CHIOSSOME, D.C.; ORNER, D.; HIDALGO, I.; SMITH, P.L.; SARKAHIAN, A.B.; YIV, S.H.; OWEN, A.J. Enhanced intestinal absorption of an RGD peptide from water-in-oil microemulsions of different composition and particle size. J. Control. Rel., Arlington, v.34, p.109-116, 1995. 
CONSTANTINIDES, P.P. Lipid microemulsions for improving drug dissolution and oral absorption: physical and biopharmaceutical aspects. Pharm. Res., Arlington, v.12,p.1561-1572, 1995.

CONSTANTINIDES, P.P.; SCARLAT, J.P.; LANCASTER, C.; MARCELLO, J.; MARKS, G.; ELLENS, H.; SMITH, P.L. Formulation and intestinal absorption enhancement evaluation of water in oil microemulsions incorporating medium-chain glycerides. Pharm. Res., Arlington, v.11, p.1385-1390, 1994.

CORREA, M.A.; SCARPA, M.V.; FRANZINI, M.C.; OLIVEIRA, A.G. On the incorporation of the nonsteroidal anti-inflammatory naproxen into cationic $\mathrm{O} / \mathrm{W}$ microemulsions. Colloids Surf. B, Amsterdam, v.43, p.106-112, 2005.

CORREA, M.A. Incorporação de naproxeno em sistema microemulsionado: liberação in vitro e avaliação biológica. São Paulo, 1996. 146p. [Tese de Doutorado. Faculdade de Ciências Farmacêuticas, Universidade de São Paulo].

CORTESI, R.; ESPOSITO, E.; MAIETTI,A., MENEGATTI, E.; NASTRUZZI, C. Formulation study for the antitumor drugs camptothecin: Lipossomes, micellar solution and a microemulsion. Int. J. Pharm., Amsterdam, v.159, p.95103, 1997.

CORTESI, R.; NASTRUZZI, C. Liposomes, micelles and microemulsion as new delivery systems for cytotoxic alkaloids. Pharm. Sci. Technol., Amsterdam, v.2, n.7, p.288-298, 1999.

CRUZ, D'J.O.; UCKUN, M.F. Gel-microemulsions as vaginal spermicidal and intravaginal drug delivery vehicles. Contraception, Stoneham, v. 64, p.113-123, 2001.

DALMORA, M.E.A.; DALMORA, S.L.; OLIVEIRA, A.G. Inclusion complex of piroxicam with b-cyclodextrin and incorporation in cationic microemulsion. In vitro drug release and in vivo topical anti-inflammatory effect. Int. J. Pharm., Amsterdam, v.222, p.45-55, 2001.

DALMORA, M.E.A.; OLIVEIRA, A.G. Inclusion complex of piroxicam with E-cyclodextrin and incorporation in hexadecyltrimethylammonium bromide based microemulsion. Int. J. Pharm., Amsterdam, v.184, p.157-164, 1999.
DALMORA, M.E.A. Interação do piroxicam com microemulsão e E-ciclodextrina: formulação liberação in vitro e avaliação biológica. Santa Maria, 1996. 147p. [Dissertação de Mestrado. Centro de Ciências da Saúde, Universidade Federal de Santa Maria].

ENGSTRÖM， S.; LJUSBERG-WAHREN, H.; GUSTAFSSON, A. Bioadhesive properties of the monoolein-water system. Pharm. Tech. Europe, Chester, v.7, p.14-17, 1995.

EZRAHI, S.; ASERIN, A.; GARTI, N. Aggregation behavior in one-phase (Winsor IV) microemulsion systems. In: KUMAR, P.; MITTAL, K.L., ed. Handkook of Microemulsion Science and Technology. New York: Marcel Dekker, 1999. cap.7, p.195-240.

FARKAS, E.; ZELKÓ, R.; TÖRÖK, Gy.; RÁCZ, I.; MARTON S. Influence of chlorhexidine species on the liquid crystalline structure of vehicle. Int. J. Pharm., Amsterdam, v.213, p.1-5, 2001.

FARKAS, E.; ZELKÓ, R.; NÉMETH, Zs.; PÁLINKÁS, J.; MARTON S.; RÁCZ, I. The effect of liquid crystalline structure on chlorhexidine diacetate release. Int. J. Pharm., Amsterdam, v.193, p.239-245, 2000.

FORMARIZ, T.P.; WANCZINSKI, B.J.; JÚNIOR-SILVA, A.A.; SCARPA, M.V.; OLIVEIRA, A.G. Biotecnologia de sistemas coloidais aplicável na otimização do efeito terapêutico de fármacos usados no tratamento do câncer. Infarma, Brasília, v.16, n.1, p.44-57, 2004.

FORMARIZ, T.P., Incorporação da doxorrubicina em microemulsões estabilizadas por fosfatidilcolina de soja e estudo da atividade antiproliferativa "in vitro" através de cultura de células. Araraquara, 2004. 199p. [Dissertação de Mestrado. Faculdade de Ciências Farmacêuticas, Universidade Estadual Paulista].

FRIBERG, S.E.; BOTHOREL, P. Microemulsions: Structure and dinamics. New York: CRC, 1988. 219p.

GABBOUN, N.H.; NAJIB, N.M.; IBRAHIM, H.G.; ASSAF, S. Release of salicylic acid and diclofenac acid salts from isotropic and anisotropic nonionic surfactant sustems across rat skin. Int. J. Pharm., Amsterdam, v.212, p.73-80, 2001. 
GARTI, N.; ASERIN, A. Pharmaceutical emulsions, double emulsion and microemulsion. In: . Drug and the pharmaceutical science. New York: Marcel Dekker, 1996. v.73, cap.15, p.412-519.

GASCO, M.R.; PATARINO, F.; LATTANZI, F. Long-acting delivery systems for peptides: reduced plasma testosterone levels in male rats after a single injection. Int. J. Pharm., Amsterdam, v.62, p.119-123, 1990.

HYDE, S.T. Identification of lyotropic liquid crystalline mesophases. In: HOLMBERG, K., ed. Handbook of Applied Surface and Colloid Chemistry. New York: John Wiley \& Sons, 2001. cap.16, p.299-332.

HO, H.; HSIAO, C.C.; SHEU, M.T. Preparation of microemulsions using polyglycerol fatty acid esters as surfactant for the delivery of protein drugs. J. Pharm. Sci., Washington, v.85, p.138-143, 1996.

HOU, M.J.; KIM, M.; SHAH, D.O. A light-scattering study of on the droplet size and interdroplet interaction in microemulsions of AOT - oil - water system. J. Coll. Int. Sci., London, v.123, p.398-412, 1988.

IBRAHIM, H.G.; SALLAM, E.; TAKIEDDIN, M.; HABBOUB, M. Effects of solutes characteristics and concentration on a lyotropic liquid crystal: solute-induced phase change. Pharm. Res., Arlington, v.10, p.737-742, 1993.

ITOH, K.; MATSUI, S.; TOZUKA, Y.; OGUCHI, T.; YAMAMOTO, K. Improvement of physicochemical properties of N-4472. Part II: characterization of N-4472 microemulsion and the enhanced oral absorption. Int. J. Pharm., Amsterdam, v.246, p.75-83, 2002.

KAHLWEIT, M.; STREY, R.; HAASE, D. How to study microemulsions. J. Coll. Int. Sci, London, v.118, p.436453, 1987.

KREILGAARD, M. Influence of microemulsions on cutaneous drug delivery. Adv. Drug Del. Rev., Arlington, v.54, p.77-98, 2002.

KUNEIDA, H.; UMIZU, G.; YAMAGUGHI, Y. Mixing effect of polyoxyethylene-type nonionic surfactants on the lipid crystalline structures. J. Coll. Int. Sci., London, v.218, p.88-96, 1999.
LANGEVIN, D. Microemulsions. Acc. Chem. Res., Washington, v.21, p.255-260, 1988.

LAWRENCE, M.J. Microemulsions as Drug Delivery Vehicles. Curr. Op. Coll. Int. Sci., Gaithersburg, v.1, p.826-832, 1996.

LAWRENCE, M.J; REES, D.G. Microemulsions-based as novel drug delivery systems. Adv. Drug Del. Rev., Arlington, v.45, p.89-121, 2000.

LEE, J.; KELLAWAY, I.W. Buccal permeation of [D-Ala ${ }^{2}$, D-Leu ${ }^{5}$ ]enkephalin from liquid crystalline phases of glyceryl monooleate. Int. J. Pharm., Amsterdam, v.195, p.35-38, 2000.

LEE, M.J.; LEE, M.H.; SHIN, C.K. Inverse targeting of drugs to reticuloendothelial system-rich organs by lipid microemulsion emulsified with poloxamer 338. Int. J. Pharm., Amsterdam, v.113, p.175-187, 1995.

LI L.; NANDI, I.; KIM, H.K. Development of an ethyl laurate-based microemulsion for rapid-onset intranasal delivery of diazepam. Int. J. Pharm., Amsterdam, v.237, p.77-85, 2002.

LINDMAN, B.; FRIBERG, S.E. Microemulsions - A historical overview. In: KUMAR, P.; MITTAL, K.L., eds. Handbook of microemulsion science and technology. Basel, New York: Dekker, 1999. p.1-12.

LUNDBERG, B.B.; MORTIMER, B.C.; REDGRAVE, T.G. Submicron lipid emulsions containing amphipathic polyethylene glycol for use as drug-carriers with prolonged circulation time. Int. J. Pharm., Amsterdam, v.134, p.119-127, 1996.

MARANHÃO, R.C.; HUNGRIA, V.T.M.; CHAMONE, D.A.F.; CHIANOTE, C.S. Microemulsions that bind to LDL receptors (LDE) as vehicles for anticancer drugs: A phase I/Phase II study of complex LDE-carmustina in multiple myeloma patients. Int. J. Hematol., Amsterdam, v.64,p.127, 1996.

MARANHÃO, R.C.; GRAZIANI, S.R.; BRANDIZZI, L.L.; COELHO, I.J.; OLIVIRA, M.A.; HEGG, R.; PINOTTI, J.A.; PILLEGGI, F.; MENEGUETTI, C.Amicroemulsion resembling low-density protein may be used as a vehicle of chemotherapeutic agents in mammary carcinoma. Can.J. Infect. Dis., Winnipeg, v.6, p.227c, 1995. 
MIYAZAKI, S.; YOKOUCHI, D.; NAKAMURA, T.; HASHIGUSHI, N.; HOU, W.M.; TAKADA, M. Pluronic F-127 gels as a novel vehicle for rectal administration of indomethacin. Chem. Pharm. Bull., Tokyo, v.34,p.1801-1808, 1986.

MITRA, S.; GAUR, U.; GHOSH, P.C.; MAITRA, N.A. Tumor targeted delivery of encapsulated dextrandoxorubicin conjugate using chitosan nanoparticles as carrier. J. Control. Rel., Arlington, v.74, p.317-323, 2001.

MITTAL, L.K. Handbook of microemulsion science and technology. New Yok: Promod Kumar, 1999. p.1-13.

MO, C.;ZHONG, M.;ZHONG, Q. Investigation of struture and structural transition in microemulsion systems of sodium dodecyl sulfonate $+n$-heptane $+n$-butanol + water by cyclic voltammetric and electrical conductivity measurements. $J$. Elect. Chem., Amsterdam, v.493, p.100-107, 2000.

MULLER-GOYMANN, C.C.; FRANK, S.G. Interaction of lidocaine and lidocaine $\mathrm{HCl}$ with the liquid crystal structure of topical preparation. Int. J. Pharm., Amsterdam, v.29, p.147-159, 1986.

NIELSEN, L.S.; SCHUBERT, L.; HANSEN, J. Bioadhesive drug delivery systems I. Characterisation of mucoadhesive properties of systems based on glyceryl mono-oleate and glyceryl mono-linoleato. Eur. J. Pharm. Sci., Arlington, v.6, p.231-239, 1998.

NORLING, T.; LADING, P.; ENGSTRON, S.; LARSSON, K.; KROG, N.; NISSEN, S.S. Formulation of a drug delivery system based on a mixture of monoglycerides and triglycerides for use in the treatment of periodontal disease. J. Clin. Periodontol., Copenhagen, v.19, p.687-692, 1992.

OLIVEIRA,A.G.; SCARPA, M.V.; CORREA, M.A.; CERA, L.F.R.; FORMARIZ, T.P. Microemulsões: estrutura e aplicações como sistema de liberação de fármacos. Quim. Nova, São Paulo, v.27, p.131-138, 2004.

OLIVEIRA, A.G.; SCARPA, M.V. Microemulsões I: fundamentos teóricos da formação do sistema microemulsionado.Infarma, Brasília, v.1,n.8, p.73-79, 2001.

OLIVEIRA, A.G.; SCARPA, M.V.; CHAIMOVICH, H. Effect of hexadecyltrimethylammonium bromide-based microemulsions on the rate of decomposition of the betalactam antibiotic cephaclor. J. Pharm. Sci., Washington, v.86, p.616-620, 1997.
OLIVEIRA, A.G. Efeitos cinéticos e mecanismos de micelas e microemulsões nas reações de decomposição de a-aminofenil penicilinas $e$ cefalosporinas. Araraquara, 1997. 160p. [Tese de Livredocência. Faculdade de Ciências Farmacêuticas, Universidade Estadual Paulista].

ORTHABER, D.; GLATTER, O. Synthetic phospholipid analogs: a structural invertigation with scattering methods. Chem. Physics Lipid,Amsterdam, v.107, p.179-189, 2000.

OWENS, D.M.; BAILLIE, G.; HALBERT, W.G. Physicochemical properties of microemulsion analogues of low density lipoprotein containing amphiphatic apoprotein B receptor sequences. Int. J. Pharm., Amsterdam, v.228, p.109-117, 2001.

PARK, M.K.; KIM, K.C. Preparation and evaluation of flubiprofen-loaded microemulsion for parenteral delivery. Int. J. Pharm., Amsterdam, v.181, p.173-179, 1999.

RITSCHEL, W.A. Microemulsion for improved peptide absorption from the gastrointestinal tract. Meth. Find. Exp. Clin. Pharmacol., Barcelona, v.13, p.205-220, 1991.

ROSANO, H.L. Microemulsions. J. Soc. Cosmet. Chem., Pontiac, v.25, p.609-619, 1974.

SARCIAUX, J.M.; ACAR, L.; SADO, P.A. Using microemulsion formulations for delivery of therapeutic peptides. Int. J. Pharm., Amsterdam, v.120, p.127-136, 1995.

SCHRAMM, G. A practical approach to rheology and rheometry. $3^{\text {rd }}$.ed. Karlsruhe: ThermoHaake, 2002, 291p.

SCOOT, H. Reology. In: REMINGTON, J.P., ed. The science and practice of pharmacy. $20^{\circ} \mathrm{sd}$ ed. Philadelphia: Editora Lippincoot, 2000. cap.23, p.335-335.

SILVA, C.R.S.H. Estudo de sistemas microemulsionados para aplicação na extração e formulações de própolis. Natal, 2001. 106p. [Dissertação de Mestrado. Instituto de Química, Universidade Federal do Rio Grande do Norte].

SIROTTI, C.; COCEANI, N.; COLOMBO, I.; LAPASIN, R.; GRAS, M. Modeling of drug release from microemulsions: a peculiar case. J. Membrane Sci., Amsterdam, v.204, p.401-412, 2002. 
SHAH, J.S.; SADHALE, Y.; CHILUKURI, D.M. Cubic phase gels as drug delivery systems. Adv. Drug Deliv. Rev., Arlington, v. 47 p. 229-250, 2001.

TROTTA, M. Influence of phase transformation on indomethacin release from microemulsions. J. Control. Rel., Arlington, v.60, p.399-405, 1999.

TROTTA, M.; PATTARINO, F.; IGNONI, T. Stability of drug-carrier emulsions containing phosphatidylcholine mixtures. Eur. J. Pharm. Biopharm., Arlington, v.53, p.2063-208, 2002.

URBAN, M.C.C. Desenvolvimento de sistemas de liberação micro e nanoestruturados para administração cutânea do acetato de dexametasona. Araraquara, 2004. 137p. [Dissertação de Mestrado. Faculdade de Ciências Farmacêuticas, Universidade Estadual Paulista].
WARGAFTIG, T.N. Desenvolvimento de microemulsões lipídicas estabilizadas com fosfatidilcolina de soja para veiculação de vacina de DNA contra tuberculose. Araraquara, 2000, 90p. [Dissertação de Mestrado. Faculdade de Ciências Farmacêuticas, Universidade Estadual Paulista].

WINSOR, P.A. Hidrotody, solubilization, and related emulsification processes. J. Chem. Trans. Faraday Soc., London, v.44, n.1, p.376-398, 1948.

WOOD, J.H. Reologia farmacêutica. In: LACHMAN, L.; LIBERMAN, H.; KANIG, L.J., ed. Teoria e prática na indústria farmacêutica. 3 ed. Philadelphia: Lea \& Febiger, 1986. v.1, cap.6, p.210-253.

WYATT, D.M.; DORSCHEL, D.A. Cubic-phase delivery system composed of glyceryl monooleate and water for sustained release of water-soluble drugs. Pharm. Technol., Duluth, v.16, p.116, 118, 120, 122, 130, 1992.

Recebido para publicação em 21 de março de 2005. Aceito para publicação em 12 de julho de 2005. 